\title{
IL PUNTO DI VISTA DEGLI "INASCOLTATI". LE NARRAZIONI DELLE DISEGUAGLIANZE SOCIALI DEI GIOVANI DI MESTRE E MARGHERA, NELLA CITTÀ DI VENEZIA
}

\author{
Francesca Campomori ${ }^{*}$, Francesco Della Puppa ${ }^{* *}$ e Giulia Storato***
}

Abstract. La crisi economica ha inasprito le diseguaglianze sociali, colpendo alcune categorie sociali, tra le quali i giovani: il loro tasso di disoccupazione è aumentato e gli investimenti sociali a loro dedicati sono stati ridimensionati. Se tale quadro strutturale è stato ben approfondito dalla letteratura sociologica, i contributi sulle percezioni dei giovani circa le diseguaglianze sociali sono ancora piuttosto rari. Il presente articolo analizza le narrazioni relative alle diseguaglianze sociali di giovani con diversi background, ma residenti nelle medesime municipalità (Mestre e Marghera) della Città di Venezia. Gli autori mostrano come tali narrazioni siano modellate sulla base dell'intersezione tra classe sociale, appartenenza "etnico-razziale" e nazionale, genere, età e collocazione spaziale nei quartieri dei giovani intervistati.

Parole chiave: Diseguaglianze Sociali; Giovani; Narrazioni; Venezia; Intersezionalità.

\section{The Point of View of the "Unheard". Narratives on Social Inequalities of Young People in Mestre and Marghera, in the City of Venice}

Abstract. The economic crisis increased social inequalities, affecting, in particular, some social subjects, such as young people: their unemployment rate increased and the social investment for them have been further cut. If this structural framework has been deepened in the sociological literature, the insights on the perceptions of young people on inequalities are much more rare. This article analyses the narratives on social inequalities of young people with different backgrounds, but residing in the same municipalities (Mestre and Marghera) of the City of Venice. The authors show how their narratives on inequalities are shaped on the basis of the intersection between social class, "ethnicity" and national background, gender, age and spatial location in the neighbourhoods.

Keywords: Social Inequalities; Young People; Narratives; Venice; Intersectionality.

*Professore associato, Dipartimento di Filosofia e Beni Culturali, Università Ca' Foscari di Venezia.e-mail: francesca.campomori@unive.it.

**Ricercatore, Dipartimento di Filosofia e Beni Culturali Università Ca' Foscari di Venezia. e-mail francesco.dellapuppa@unive.it.

***Borsista di ricerca, Dipartimento di Filosofia e Beni Culturali Università Ca' Foscari di Venezia.e-mail: fgiulia.storato@unive.it.

Sociologia e Politiche Sociali, vol. 22, 3/2019 pp. 215-241.

Paper ricevuto in data: 16/01/2019 - Paper accettato in data: 08/01/2020.

ISSN 1591-2027; ISSNe 1972-511 


\section{Introduzione}

Le diseguaglianze che attraversano la società hanno tradizionalmente costituito un tema centrale della riflessione sociologica (Gallino 2000; Zanfrini 2011) che ha analizzato come queste si articolano in qualsiasi ambito della società.

Fra questi, il mercato del lavoro svolge un ruolo chiave tanto nella produzione, quanto nella manifestazione delle disparità sociali (Barbieri e Fullin 2014; Gosetti 2007). Oltre ai i lavoratori over50 in cerca di un reinserimento (Jubany and Perocco 2015) e gli immigrati (Saraceno et al. 2012), tra i soggetti sociali che si trovano in condizione di maggior vulnerabilità conseguentemente ai cambiamenti in atto nel mondo del lavoro troviamo i giovani (Azzolini et al. 2011; Bello and Cuzzocrea 2018; Rebughini et al. 2017; Schizzerotto et al. 2011).

Le diseguaglianze lavorative si ripercuotono, ovviamente, anche sulle altre sfere del vivere sociale (Azzolini et al. 2013), in primis quella abitativa e residenziale. La sociologia urbana si è da sempre concentrata sulla stratificazione residenziale delle diverse fasce della popolazione, sugli eventuali fenomeni di segregazione abitativa e sulle sue conseguenze rispetto alla riproduzione delle diseguaglianze sociali di cui può essere essa stessa prodotto (Güentner et al. 2018).

Negli Stati Uniti, hanno iniziato a manifestarsi negli anni '20 con la migrazione interna della popolazione "afro-americana" verso i centri industriali del nord (Denton and Massey 1993; Wacquant 1995; 2007) e il trasferimento delle classi medie bianche verso le periferie residenziali che si stavano sviluppando. In Europa, la segregazione ha assunto dimensioni ridotte, in virtù di un welfare state più forte. I grandi complessi residenziali di edilizia popolare non erano pensati esclusivamente per le classi popolari, ma prevedevano la mixité con i ceti impiegatizi (Bricocoli e Padovani 2006; Oberti 2006). A partire dagli anni ' 70 , però, si caratterizzano sempre più per la concentrazione abitativa degli strati subalterni, con ampia sovrarappresentanza degli immigrati (Agustoni e Alietti 2009). Un'analisi classica rispetto all'intreccio tra diseguaglianze e accesso stratificato ad abitazione e spazi urbani è fornita da Pierre Bourdieu (1993), secondo cui le diseguaglianze sociali si riflettono in quelle spaziali e viceversa (Marrone 2012). Analogamente, le possibilità di mobilità territoriale si rifletterebbero sulle possibilità di mobilità sociale (Turner 1994). Ecco che, quindi, senza per questo negare l'importanza delle determinanti strutturali, un ruolo cruciale lo svolge la dimensione locale e, nei contesti urbani, i quartieri e le infrastrutture sociali in essi presenti (o assenti) (Güentner et al. 2018) che costituirebbero, cioè, delle "strutture strutturate e strutturanti" (Bourdieu 1979), attraverso le quali un determinato ordine sociale viene percepito e riprodotto. Gli effetti positivi o negativi che un quartiere ha sulle possibilità di vita dei suoi residenti, i cosiddetti "effetti di luogo" (Bourdieu, 1983) o "effetti di zona" (Atkinson e Kintrea 2004; Sampson et al. 2002) o, ancora, neighborhood effects (Wilson 1987), sono di difficile analisi empirica, ma sono fortemente tenuti in considerazione dalle politiche urbane dagli anni ' 80 in poi, soprattutto nei Paesi dell'Europa Nord-occidentale. 
I quartieri costituiscono delle arene di grande rilevanza per la vita quotidiana degli individui e, di conseguenza, per la loro costruzione e l'interiorizzazione di specifici habitus (Bourdieu 1993): non è possibile evitare l'immediatezza del quartiere appena varcata la soglia di casa (Parkes and Connolly 2011; Sykes e Kuyper 2009), in particolare per gli adolescenti e i giovani, soggetti con limitate possibilità di mobilità spaziale. I quartieri e i servizi in essi presenti possono rappresentare spazi dove si sperimentano le disuguaglianze sociali - potenzialmente amplificate o contrastate -, ma è difficile individuarne empiricamente l'effetto sul comportamento e sulle prospettive di vita dei giovani. Assumere una concezione dello spazio in termini relazionali, rende possibile cogliere sia le strutture sociali che producono tali disuguaglianze sia l'agentività degli attori nel riprodurle o sfidarle (Farrugia 2015, Worth 2015; Massey 2005). In questa prospettiva, le pratiche corporee di appropriazione, autoesclusione, sconfinamento degli spazi del quartiere, sia $\mathrm{i}$ suoi processi di significazione attraverso il linguaggio possono essere interpretati come processi costitutivi di appartenenza (Low, 2003). In altre parole, il quartiere struttura ed è, allo stesso tempo, strutturato da habitus, pratiche, disposizioni, inclinazioni sedimentate dei soggetti che lo abitano e, nelle narrazioni ad esso riferite, è possibile cogliere i meccanismi di distinzione sociale e $\mathrm{i}$ processi, più o meno espliciti, di esclusione e marginalità - a loro volta espressi in termini relazionali e spaziali, ovvero in luoghi "di confine", "proibiti", "contesi", "nascosti" del quartiere stesso (Storato 2014).

Rispetto al tema specifico delle disuguaglianze, il lavoro di Alan Murie (2005) analizza approfonditamente il ruolo dei quartieri nella produzione, nella riproduzione, ma anche nell'attenuazione delle diseguaglianze, facendo riferimento alle articolazioni dell'esclusione sociale. In particolare, l'A. si sofferma sia sulle concrete opportunità offerte da una determinata porzione urbana, sia - e soprattutto sul ruolo delle rappresentazioni e percezioni degli individui ad esse relative e sulla formazione di specifici habitus, come elementi determinanti l'esperienza dell'esclusione. Le narrazioni degli individui appartenenti ai gruppi sociali più vulnerabili rispetto alla propria condizione occupazionale e residenziale, infatti, possono essere determinanti nel configurare concretamente le loro opportunità e la loro agency (Shildrick 2006). In questa sede, dunque, ci si focalizzerà proprio sulle narrazioni relative alle diseguaglianze di uno dei gruppi sociali in condizione di maggior vulnerabilità socio-economica - i giovani tra i 16 e i 25 anni -, a partire da quelle relative al lavoro, ma comprendendo anche quelle legate all'utilizzo degli spazi urbani nella vita quotidiana, in quanto compartecipano alla concreta strutturazione delle loro traiettorie sociali. Per fare ciò, si assumerà una prospettiva intersezionale, ritenendola particolarmente funzionale al tema e agli obiettivi presentati, ossia la messa in luce delle diverse articolazioni dell'esperienza quotidiana delle diseguaglianze degli individui. Coniato da Crenshaw $(1989$; 1991), il termine "intersezionalità" esprime, nelle ragioni che hanno portato alla sua stessa nascita, un forte legame con il tema dell'invisibilità e delle disuguaglianze. Sviluppato e ampiamente discusso all'interno del dibattito femminista (Walby et al. 2012; Davis 2008; Yuval-Davis 2006;), tale approccio offre strumenti per un'analisi critica e 
stratificata dei processi di costruzione sociale delle appartenenze e delle politiche di appartenenza (Yuval-Davis 2011). In particolare, esso afferma come, nell'esplorare le esperienze di vita quotidiana degli individui, sia necessario tener conto di come forme di appartenenza ascritta e divisioni sociali (età, genere, classe, origine "etnico-razziale", abilità, etc.) interagiscano, costruendosi reciprocamente (Anthias 2008).

Adottare questa prospettiva implica quindi, analizzare le narrazioni dei giovani, non solo riconoscendo la peculiarità delle loro esperienze in quanto giovani, ma anche esplorare come l'appartenenza alla medesima generazione possa essere esperita diversamente nell'intersezione con il quartiere in cui risiedono, la loro la collocazione di classe, l'appartenenza di genere e l'origine "etnico-nazionale" (Colombo and Rebughini 2017; Mancini 2016), mettendo in luce possibili stratificazioni interne.

Nello specifico, ci si concentrerà sui giovani residenti nelle municipalità di Mestre e Marghera - quartieri costituenti la componente principale della "terraferma" della Città di Venezia.

Dopo un breve approfondimento sulla metodologia adottata, l'articolo avanzerà una ricostruzione storico-sociale dei contesti analizzati al fine di investigare l'origine delle diseguaglianze sociali che li caratterizzano e che, nel particolare caso di Marghera, ne fanno il quartiere socialmente più fragile del comune di Venezia e uno dei più fragili dell'intero Veneto. Seguiranno le sezioni principali in cui verranno presentati i risultati empirici relativi alle narrazioni dei giovani circa le diseguaglianze sociali rispetto alla loro residenza in specifiche aree dei quartieri, alla collocazione di classe delle loro famiglie di origine, all'appartenenza di genere; all'origine nazionale; al posizionamento generazionale.

\section{Nota metodologica}

Il presente contributo è frutto di un più ampio progetto di ricerca, intitolato $C I-$ TISPYCE. Combating inequalities through innovative social practices of, and for, young people in cities across Europe, finanziato dalla Commissione Europea all'interno del VII Programma Quadro e condotto, tra il 2013 e il 2016, con un approccio comparativo in dieci Paesi dell'Unione europea: Italia, Spagna, Polonia, Grecia, Regno Unito, Bulgaria, Germania, Paesi Bassi, Repubblica Ceca, Svezia (Güentner et al. 2018). Il progetto ha prima prodotto una ricognizione delle diseguaglianze sociali relativamente alla fascia giovanile, nelle dieci città oggetto dello studio $^{1}$; successivamente, ha analizzato le pratiche sociali innovative emerse in alcuni quartieri di queste città (Campomori e Della Puppa 2014; 2017; Carrosio 2016; Villa 2016).

${ }^{1}$ Amburgo, Atene, Barcellona, Birmingham, Brno, Cracovia, Malmö, Rotterdam, Sofia, Venezia. 
I dati utilizzati per la stesura del presente articolo sono stati raccolti attraverso la raccolta di 37 interviste in profondità e la conduzione 3 focus group con giovani tra i 16 e i 25 anni, di origine autoctona o immigrata - questi ultimi tutti dal Bangladesh, una delle collettività immigrate più giovane e rappresentativa nei contesti analizzati -, residenti nei quartieri analizzati.

Gli intervistati sono stati contattati soprattutto grazie all'intermediazione di educatori di strada ed operatori di servizi presenti a Mestre e Marghera, ma anche attraverso associazioni giovanili in cui gli stessi intervistati sono coinvolti e mediante la frequentazione di spazi pubblici urbani in cui parte degli intervistati trascorre il tempo libero (parchi, piazze, campi sportivi, sale prove pubbliche, parrocchie). Sono, così, stati intervistati giovani coinvolti in attività religiose e parrocchiali, membri dei comitati civici e di quartiere, scout, utenti dei servizi e frequentatori di luoghi di aggregazione municipale. Tra gli intervistati ci sono lavoratori, studenti medi e universitari, studenti-lavoratori, lavoratori-studenti, giovani disoccupati in cerca di lavoro, "Neet". Per aumentare l'eterogeneità del gruppo di intervistati, infatti, si è dato avvio a diverse snowball (Cardano, 2003; 2011; La Mendola, 2009; Silverman, 2007; 2008) a partire dagli stessi giovani intervistati, ma anche coinvolgendo ulteriori giovani con cui i entravamo in contatto durante le interviste e la frequentazione di spazi pubblici condivisi.

La vicinanza generazionale di alcuni membri dell'equipe di ricerca ai soggetti al centro dell'indagine o agli operatori con cui i giovani stessi hanno avuto modo di confrontarsi più volte in passato (educatori e operatori di strada in primis) ha facilitato il contatto con gli intervistati e ha permesso l'instaurazione di relazioni di fiducia e confidenza - al punto da rischiare di renderne complicata la gestione e di rendere difficile un chiaro mantenimento/definizione dei ruoli.

L'approccio all'intervista è stato di tipo "dialogico" (La Mendola, 2009). Tale prospettiva ha previsto la rielaborazione e l'operativizzazione della traccia tramite domande "generative" (Becker, 2007), che facciano emergere, cioè, processi più che informazioni: quesiti che favoriscono il fluire delle esperienze, più che l'esperienza puntuale, partendo, però, da episodi concreti e per mezzo di rilanci (anche cenni del capo, espressioni di assenso, silenzi...).

I focus group sono stati utilizzati per approfondire o illuminare specifici temi che risultavano poco chiari da una pre-analisi dei dati costruiti attraverso le interviste e sono stati coinvolti quasi sempre giovani precedentemente intervistati individualmente. Per concludere, va sottolineato l'ampio e utile utilizzo dei social network come strumento di creazione e mantenimento dei contatti con i giovani coinvolti nell'indagine, di organizzazione degli appuntamenti con gli stessi. I nomi degli intervistati, riportati in calce alle interviste, sono fittizi. 


\section{Il contesto di indagine}

3.1 Mestre: alla ricerca di un'identità, tra gli errori del passato e le criticità del presente

Nel primo ventennio del secolo scorso, Mestre, allora Comune autonomo e separato da Venezia, aveva cominciato a strutturarsi come città attraverso la costruzione delle principali infrastrutture, servizi urbani e dei primi stabilimenti industriali (Casarin e Saccà, 2009). Nel 1926, il destino di Mestre è stato legato strettamente a quello di Venezia, con un decreto ministeriale che ne stabiliva l'annessione, al fine di garantirle più ampie opportunità per una sua espansione industriale e residenziale. Prende forma, così, l'idea di una "Grande Venezia": una città più estesa rispetto alla sola laguna, a comprendere anche territori della terraferma, in cui Mestre avrebbe avuto una funzione residenziale e Marghera un ruolo industriale. In pochi decenni, Mestre è diventata da cittadina di piccole dimensioni ad agglomerato urbano e industriale medio-grande, con circa 90.000 residenti $^{2}$ e molti domiciliati (Ibidem).

La parte più consistente del quartiere fu costruita dopo la Seconda guerra mondiale, quando molti abitanti della Venezia lagunare iniziarono a trasferirsi a Mestre. Al contempo, Mestre aveva cominciato ad accogliere anche i residenti provenienti dalla provincia veneziana e da quelle adiacenti, attratti dalle opportunità di inserimento lavorativo e abitativo offerte. Ciò ha comportato una crescita tanto rapida quanto disorganica, caratterizzata da fenomeni di speculazione edilizia, che ha finito per generare un agglomerato sovrappopolato, urbanisticamente disordinato ed esteticamente brutto. Pur senza particolari fenomeni concentrativi, sono sorti, così, in diverse aree del quartiere, enormi complessi abitativi di tipo "popolare", oggi in condizioni precarie.

Nell'ultimo passaggio di secolo, Mestre ha iniziato ad attraversare una fase di transizione che l'ha spinta a interrogarsi sulla propria identità e sulle trasformazioni che l'hanno investita. Rispetto alla Venezia "lagunare", infatti, Mestre si è sempre - ed è sempre stata - percepita come una parte di città a sé. Se, da un lato, il turismo di massa ha profondamente trasformato il centro storico di Venezia, rendendo sempre più difficile e dispendiosa la vita dei residenti, dall'altro lato, le attività socio-economiche, le istituzioni politiche e amministrative e, non ultimo, le persone si sono trasferite - e si stanno trasferendo - verso la "terraferma" (Ibidem).

Il presente di Mestre, inoltre, è segnato da quello di Porto Marghera: dal suo sviluppo, prima, e dal suo declino, poi; dalle ampie possibilità di inserimento lavorativo iniziali e, negli ultimi decenni, della crescente disoccupazione. Inevitabil-

${ }^{2}$ In soli venti anni Mestre ha decuplicato la propria popolazione (da 20.000 a 200.000 abitanti), se si considera il continuum urbanistico-residenziale che ha formato con i centri circostanti. Con il toponimo "Mestre", infatti, nel linguaggio comune si designa spesso tutta la terraferma veneziana, dove risiedono due terzi della popolazione dell'intero Comune di Venezia. 
mente, ciò ha avuto pesanti effetti anche sulle condizioni di vita dei residenti, portando a un aumento delle famiglie in situazione di fragilità socio-economica, seguite dai servizi sociali, di cui il $40 \%$ di origine immigrata. A Mestre, infatti, anche se con cifre inferiori rispetto a Marghera, il tasso di residenti immigrati è superiore a quello del resto di Venezia nel suo complesso con aree che presentano percentuali di residenti immigrati tra il 20 e il 30\%. Secondo il Servizio Statistico e Ricerca della Città di Venezia, in generale, nel 2000, la popolazione immigrata costituiva il $2 \%$ dei residenti a Venezia, per arrivare al $10 \%$ nel 2010 e al $12,5 \%$ nel 2015. A Mestre, invece, negli stessi anni, queste percentuali salivano rispettivamente al $2,7 \%$, al $14,2 \%$ e al $18,7 \%$.

Nel 2014, il numero di numero di persone di età compresa tra 0-21 anni in carico ai servizi è stato 306 (1'1,6\% della popolazione nella fascia di età di riferimento) e più della metà di loro hanno superato i 14 anni. L'intervento più diffuso è l'erogazione di un contributo economico, seguito dal sostegno educativo domiciliare. Infine, va rilevato l'alto e diffuso consumo di sostanze, in particolare eroina, da parte della componente più giovane. Tale fenomeno, già molto grave negli anni ' 80 , sembrerebbe in crescita esponenziale tra i giovanissimi, nell'ultimo quinquennio (Sevizi Sociali di Mestre, 2014).

\subsection{Disagio e resilienza: Marghera e le sue ferite}

L'idea di dare vita a Porto Marghera iniziò a prendere forma nei primi del '900, quando divenne evidente che gli spazi del porto di Venezia erano insufficienti per gestire i traffici che su di esso gravitavano. Nel 1917, un decreto regio stabilì la nascita di Marghera come parte del Comune. La zona era quasi completamente vuota e si iniziarono a costruire un porto e un sistema viario. Nel 1922, furono inaugurate le prime fabbriche e fu lanciato il progetto per la realizzazione del quartiere urbano, che ambiva a configurarsi come una "Città Giardino" (Barizza, 2000; Barizza e Cesco, 2007). Marghera, cioè, sarebbe dovuta essere composta da una zona industriale e da un centro residenziale con molte aree verdi. Nel corso della Seconda guerra mondiale, tuttavia, l'area fu ripetutamente bombardata e nella ricostruzione post-bellica l'iniziale progetto fu accantonato (Ibidem). Dagli anni ' 50 fino alla fine degli anni ' 70 , al contrario, lo sviluppo dell'area industriale fu impetuoso, coerentemente la crescita urbanistica che caratterizzò l'Italia nei cosiddetti Trente Glorieuses. Furono costruite molte industrie, in primis, il petrolchimico e i cantieri navali (con la Fincantieri ${ }^{3}$, uno dei più importanti complessi navalcantieristici del mondo). Marghera ha assunto, così, le caratteristiche di un quartiere operaio, compromesso da un intenso inquinamento a lungo ignorato.

Nel 1971, le industrie di Porto Marghera occupavano 38.500 lavoratori, principalmente nel petrolchimico - uno dei più importanti in Europa - e il 14\% di loro era residente nel quartiere. Negli stessi anni la popolazione aumentò vertiginosamente, passando dai 7.561 abitanti del 1945 ai 27.619 del 1965, comportando uno

\footnotetext{
${ }^{3}$ All'oggi, presenta un fatturato di 2,4 miliardi di euro e 10.000 dipendenti.
} 
sviluppo edilizio rapido, ma di scarsa qualità e poco pianificato, specialmente nella parte meridionale a ridosso dell'area industriale. La crisi energetica e la stagnazione economico-industriale degli anni ' 70 non hanno risparmiato Marghera: i livelli occupazionali hanno subito un fortissimo ridimensionamento e, di conseguenza, anche il tasso di residenti (Ibidem).

Oggi, quindi, Marghera si configura come un quartiere "popolare", con un livello di istruzione più basso rispetto al resto della città e con il più alto tasso di residenti immigrati ( $20 \%$ rispetto al $12 \%$ della Città) e di giovani: il Servizio Statistico e Ricerca della Città di Venezia registra la più alta percentuale di residenti nelle fascia 0-14 (nel 2010, 12,8\% rispetto all' $11,9 \%$ della Città) e nella fascia 15 24 (9,3\% rispetto all' $8 \%)$.

La parte settentrionale del quartiere conserva alcuni aspetti del progetto di "Città Giardino", con molte aree verdi, case basse mono o bi-familiari con giardino, una piazza centrale. La parte meridionale, adiacente all'area industriale, invece, è quella che risente maggiormente delle conseguenze della sua storia travagliata e presenta abitazioni fatiscenti in condizioni igieniche precarie, al punto che per alcune è previsto un piano di demolizione con conseguente spostamento degli inquilini in altre aree.

Un'ulteriore eredità del passato che grava sul presente di "Marghera sud" risale alla decisione, presa dall'amministrazione locale in epoca fascista, di concentrare in questo segmento le componenti della popolazione socio-economicamente più vulnerabili. Ciò ha comportato la concentrazione. in un'area territorialmente molto ristretta, di forti criticità che, riverberandosi nel tempo, si sono riprodotte fino ai giorni nostri. Oggi, ad esempio, "Marghera sud" costituisce il centro principale del traffico di droga nella provincia veneziana e nelle aree adiacenti - con ovvie ricadute su ampie fasce di giovani e giovanissimi.

Va poi menzionata una specifica porzione del quartiere, la "Cita", a ridosso della stazione ferroviaria. Si tratta di una vasta area con quasi un migliaio di appartamenti di housing sociale all'interno di "condomini alveare" eretti negli anni del cosiddetto "boom economico" (Marzadro 2010). In origine, la Cita era stata progettata per il ceto impiegatizio benestante, ma la sua composizione sociodemografica è cambiata radicalmente quando, sul finire degli anni ' 70 , uno dei suoi palazzoni ha accolto le famiglie provenienti dalle abitazioni che da lì a poco sarebbero state demolite a Marghera sud: nuclei familiari con vulnerabilità economiche e sociali. Oggi, la Cita è contraddistinta anche dalla più alta densità di residenti immigrati $(33 \%)$ rispetto al resto del quartiere (Ibidem). Tale dato va inquadrato nella cornice generale dell'immigrazione a Marghera, in cui, come accennato, tanto in termini percentuali quanto in termini assoluti, la quota di residenti immigrati è sempre stata nettamente superiore alla media in tutta la città. Se, nel 2000, la componente immigrata costitutiva il $2 \%$ della popolazione complessiva della Città di Venezia, per arrivare al 10\% nel 2010 e al 12,5\% nel 2015, a Marghera, negli stessi anni, questa componente dei residenti costituiva rispettivamente il $2,6 \%$, il $17,8 \%$ e il $20,6 \%$. 
Marghera presenta anche i tassi più elevati di nuclei familiari presi in carico dai servizi sociali (o che da questi ricevono contributi di diverso tipo) e di bambini allontanati dalle famiglie di origine dell'interno Comune di Venezia. Il Rapporto Annuale dei Servizi Sociali di Marghera per il 2012, infatti, segnala una crescita continua delle prese in carico di soggetti sotto i 22 anni: dai 165 casi del 2006, ai 285 del 2012. La stessa tendenza si registra per quello che riguarda i nuclei genitoriali sostenuti dai servizi: dai 137 casi, nel 2006, ai 228, nel 2012.

\section{Le dimensioni delle diseguaglianze percepite dai giovani}

La ricerca ha permesso di delineare le direttrici attorno alle quali i giovani percepiscono strutturarsi le diseguaglianze: la collocazione abitativa in specifiche aree dei quartieri rispetto ad altre; l'appartenenza di classe delle famiglie di origine; il genere; l'origine nazionale; l'appartenenza generazionale.

\subsection{La dimensione spaziale: luoghi come vincoli e opportunità}

Le testimonianze degli intervistati restituiscono l'impressione che i diversi luoghi e le diverse aree dei quartieri strutturino in maniera sostanzialmente differente le opportunità e le traiettorie dei giovani che vi abitano (Bourdieu 1993; Lefebvre, 1974; Marrone 2012). Prenderebbe forma, cioè, una stratificazione delle possibilità sociali dei giovani sulla base delle diverse zone in cui essi risiedono (Borlini 2010) Ciò si ripercuoterebbe soprattutto sui rischi di entrare in contatto con attività illegali e il prendere parte a fenomeni di devianza - sostanzialmente lo spaccio e l'assunzione di stupefacenti:

Marghera è un territorio in cui è molto facile cadere in vari tipi di tentazione e molti miei amici ci sono caduti. [...] Tutte le persone della mia età possono confermarlo $\mathrm{e}$ l'hanno vissuto direttamente o indirettamente. Adesso forse un po' meno, ma fino a pochi anni fa era molto facile trovare facilmente eroina, cocaina, hashish... [...] C'erano dinamiche di spaccio ovunque, nella compagnia di amici o fra conoscenti o subito fuori casa... Anche nel nostro gruppo di amici, infatti, molti ci sono caduti in pieno: tossicodipendenza, sostanze pesanti, eroina. [...] Se abiti qui, è molto facile caderci, in un momento all'altro, per qualsiasi motivo di difficoltà - economiche, familiari, problemi di altro tipo... - soprattutto per i più giovani. (Maurizio, 23 anni, origine autoctona, studente e lavoratore, residente a Marghera)

Nei parchi vicino a casa mia c'è un traffico enorme di eroina, un traffico gigante. Tu stai fermo lì e conti cinque persone ogni venti minuti, dalle otto di mattina alle tre di notte, subito fuori casa. (Fabio, 22 anni, origine autoctona, studente, residente a Mestre)

Al contempo, ciò si tradurrebbe - soprattutto per quanto riguarda il quartiere di Marghera - in una stigmatizzazione (Lefebvre, 1974) che condizionerebbe, in par- 
te, le opportunità di inserimento lavorativo e, soprattutto, i percorsi scolastici dei giovani, analogamente a quanto osservato nel contesto scozzese da Sykes e Kuyper (2009) e in quello australiano da Overman (2002). Secondo le narrazioni degli intervistati:

Uno che nasce a Marghera, sin da piccolo, si sente dire: "A Marghera abiti? Caspita! Ma ti trovi bene? - E facce come per dire - Come fate a trovarvi bene a Marghera ragazzi!?" [...] Essere di Marghera da un messaggio di alternatività, di essere qualcosa di altro [...] Ti dicono: "Ah, di Marghera!? C'è solo la strada con le prostitute". Cioè capisci? Così ci vedevano. $\mathrm{O}$ "Marghera? Piena di immigrati, piena di Bangladesh" O ancora: "Quando prendo l'autobus sei barrato o il tre per andare a Marghera sento sempre puzza!" Capito? Se sei di Marghera dovresti vergognartene, [invece] a Marghera c'è l'orgoglio di essere di Marghera, noi di Marghera abbiamo un orgoglio che ti fa dire: "Io non sono come tutto il resto, sono il ribelle della società". (Maurizio, 23 anni, origine autoctona, studente e lavoratore, residente a Marghera)

Silvia: Il fatto che a Marghera non ci sia una scuola superiore mi fa pensare che sia lasciata a sé...

Andrea: Sì, a Marghera è vastissima però non ci sono scuole superiori...

Francesco: Le scuole medie sono quattro e una scuola superiore c'era, l'Edison, era un elettromeccanico, un istituto professionale, la qualifica era di officina. Era troppo grande e difficile da mantenere, quindi, hanno preferito trasferire tutto a Mestre anche se era molto frequentata. [...] Però, come dicevamo prima, quella era a Marghera e quelli di Mestre non andavano mai là: a Marghera andavano solo quelli di Marghera, quelli di Mestre non ci andavano perché si diceva che nella scuola di Marghera c'erano soggetti che comunque... Anche i professori di Mestre ti dicevano "Sei di Marghera? È dura eh?" Partono con il pregiudizio sul classico margherotto... (Focus group)

A tale narrazione di Marghera e dei suoi residenti si sovrapporrebbero, secondo gli intervistati, minori collegamenti e, quindi, minori possibilità di mobilità entro $i$ territori urbani (Atkinson e Kintrea 2004; Clark et al. 2017) che rafforzerebbero la narrazione di Marghera come "una città nella città" (Park and Burgess, 1928) o per utilizzare l'espressione di un intervistato - "una periferia della periferia" e, al contempo, l'auto-narrazione dei suoi residenti di essere "altro" da Mestre e dal resto della Città di Venezia (Atkinson e Kintrea 2004; Lefebvre 1974).

Va sottolineato, però, che la permanenza sul campo ha fatto emergere come le distanze geografiche - facilmente percorribili in bicicletta o a piedi - che separano Marghera da altre aree della Città non parrebbero di per sé un ostacolo oggettivo alla mobilità dei giovani, quanto piuttosto - come confermato dalle interviste - un ostacolo soggettivo. Prenderebbero forma, cioè, delle frontiere fisiche, ma soprattutto, simboliche (Brighenti et al. 2016; Della Puppa e Gelati,2015; Stravrides 2010), costruite anche attraverso pratiche discorsive - che inibirebbero gli spostamenti internamente ai quartieri e tra i quartieri (Güentner et al. 2018) - per quanto 
riguarda quest'ultimo aspetto, soprattutto da Mestre a Marghera più che nella direzione opposta:

Francesco: Nessuno andrà mai a piedi da Mestre a Marghera, minimo in bici se non in pullman.

Andrea: io sulla distanza in sé vedo tanti pregiudizi, soprattutto per quanto riguarda il luogo in cui vivi: se vai da un ragazzo di Mestre e dici che sei di Marghera: "Oh my god! Sei di Marghera! E di Marghera dove? - della Cita - Oh my god! Della Cita!? Si vive bene!? Ci sono rapine?" Ci sono tanti pregiudizi al di là della distanza che alla fine tra Mestre, Marghera e Venezia, tra servizi di pullman e tram, uno se la gestisce più che tranquillamente e anche se a volte non ci sono i bus, io mi faccio le camminate da Marghera a Mestre tranquillamente: sono 20 o 25 minuti.

Silvia: Dicono che la sera, da soli, non è il caso di andarci, però a me non sembra o almeno io non ho avuto esperienze brutte. Poi Marghera non esiste proprio per gli studenti: "Marghera che cos'è? Cosa ci vai a fare? Perché? Non c'è niente!"

Maurizio: Già dicono tutti che "Non c'è niente"...

Eva: Invece c'è tanto! Ho scoperto che ci sono tante cose, un gruppo di teatro dell'oppresso, il "Comitato Marghera Libera e Pensante", poi c'è il Centro Sociale "Rivolta", un sacco di associazioni, poi...[...]

Andrea: Magari le associazioni e le cose ci sono però a livello di persone... se vai in giro per Marghera e vedi sempre le stesse facce, sempre le stesse persone, non è che vedi dei giovani che vengono da altri quartieri. I ragazzi di Mestre stanno a Mestre.

Stefano: Quando avevo 15 anni ho sempre pensato: Ma perché qua a Marghera con tutti i ragazzi che siamo non c'è uno spazio per i giovani e se stiamo in piazza o in strada arriva subito la polizia, quindi o sto a casa e mi isolo e secondo me per molti è andata così oppure vado a Spinea, a Mestre... (Focus group)

Tale distanza/separazione geografico-spaziale sarebbe il risultato del prodotto combinato di vari fattori: la conformazione urbanistica dei quartieri di Mestre e Marghera; gli ostacoli architettonici che fungono da frontiere simboliche e che separano i due quartieri tra loro (la ferrovia e la stazione di Venezia Mestre), ma anche rispetto al Centro Storico della città insulare (Ponte della libertà e Strada Statale 11) e rispetto al resto del contesto territoriale adiacente (lo snodo autostradale, la ferrovia, la laguna); la citata stigmatizzazione del quartiere di Marghera.

Alla distanza/separazione geografico-spaziale descritta si accompagnerebbe, talvolta, una distanza/separazione sociale e relazionale (Gabbi 2013). Ciò sembrerebbe caratterizzare soprattutto il quartiere di Marghera, perché maggiormente isolato da Mestre e dal resto della Città a livello spaziale (per la sua posizione geografica) e a livello politico, economico, amministrativo e sociale (per la centralità - o, comunque, la peculiarità - assunta in questi ambiti da Mestre e dalla Venezia "storica") (Atkinson e Kintrea 2004).

Alla creazione di tale "separatezza" ha contribuito la genesi e l'evoluzione storica della Città e dello specifico quartiere che - soprattutto per quanto riguarda alcune sue porzioni meridionali e in un secondo momento la Cita - è stato concepito e socialmente (ma anche materialmente) costruito come luogo di destinazione di 
soggetti socialmente problematici e "unwanted", ma anche la pianificazione amministrativa della città che ha concepito le municipalità - e Marghera coincide con una di queste - come entità parzialmente autonome (Campomori e Della Puppa 2013 ; 2017). Come anticipato, le frontiere simboliche che agiscono separando tra loro i diversi quartieri agirebbero anche all'interno dei quartieri stessi (Brighenti et al. 2016; Della Puppa e Gelati 2015; Stravrides 2010). Ė emblematico il caso di un servizio dedicato ai giovani, la sala prove Monteverdi, un tempo meta e punto di aggregazione di centinaia di loro e oggi molto meno utilizzata perché decentrata di un paio di chilometri, percorribili anche a piedi, rispetto alla sede originaria:

Hanno spostato la sala prove dal centro di Mestre quasi alla periferia, non ci si va più perché bisogna prendersi un bus e farsi uno "scazzo" gigantesco per arrivare, adesso, fino a Villa Franchin... (Mirko, 22 anni, origine autoctona, studente, residente a Mestre)

\subsection{La classe sociale di appartenenza: ricadute e intersezioni}

I giovani intervistati evidenziano solo secondariamente le forme di diseguaglianze più strutturali come quelle connesse alla stratificazione sociale, da cui dipendono le altre diseguaglianze. Rispetto a ciò, il lavoro sul campo ha confermato come la diversa collocazione di classe dell'aggregato domestico a cui i giovani appartengono determini fortemente le traiettorie sociali, materiali e scolastiche dei giovani stessi (Azzolino et al. 2013; Jacobs et al. 2009; Raitano 2011; Schizzerotto et al. 2011). È il caso di Alvise che, stante l'improvvisa condizione di disoccupazione di entrambi i genitori, ha dovuto comporre tra loro tre diverse attività lavorative per soddisfare le necessità della famiglia di cui si è trovato di colpa ad essere l'unico breadwinner. Ciò ha, ovviamente, comportato ricadute sulle sue traiettorie scolastiche (Ballarino e Checchi 2006; Schizzerotto et al. 2011), portandolo ad optare per un corso serale - comunque frequentato con fatica e discontinuità:

I miei genitori lavoravano fino all'anno scorso, adesso purtroppo con la crisi sono rimasti a casa entrambi. Mio padre lavorava in un ristorante a Venezia e mia madre in un ufficio sempre a Venezia, faceva l'impiegata. Quindi lavoro in tre posti diversi: per due compagnie di trasporti a Venezia e per una pizzeria a Marghera e studio alle serali perché ho avuto un po' di "incidenti di percorso" a scuola, un po' voluti e un po' costretti. [...] Dovrei andare tutti i giorni, di fatto, però, con gli orari di lavoro e tutte le cose, è impossibile. Almeno adesso con il lavoro in pizzeria, per fortuna, è tutto in regola mi fanno dei permessi dove viene scritto che sono a lavoro e non sono in giro a bighellonare e a perdere tempo, quindi, magari, i professori cercano di venirmi incontro in questo senso: sanno che chi fa i serali generalmente ha lavoro e famiglia e, quindi, spesso ci si accorda per i compiti, le interrogazioni, si cerca di essere presente almeno per questi momenti e il resto delle ore di assenza vediamo se verrà tollerato $o$ meno. Ci sono professori che ci tengono alla presenza, però, è un po' dura riuscire a essere sempre presenti sia per gli orari che per la stanchezza... è dura. (Alvise, 21 anni, origine autoctona, studente e lavoratore, residente a Marghera) 
Ma è anche il caso di giovani appartenenti allo stesso "gruppo di frequentazione", ma con opportunità molto diverse: chi può sostenere la spesa di un alloggio in un altro contesto urbano al fine di frequentare l'università "fuori sede", pur non essendo "esattamente uno studente modello" (Francesco, 22 anni, studente, residente a Mestre) e chi è costretto a convivere con il pendolarismo quotidiano tra il quartiere di residenza e la città universitaria; chi non ha avuto "la necessità e l'interesse a cercare un lavoro perché, finché non mi laureo, comunque, ho la possibilità di farmi mantenere e vivere con i miei" (Nicola, 23 anni, studente, residente a Mestre) e chi deve conciliare impegni universitari o scolastici con quelli lavorativi (Colombo et al. 2018). È il caso dei ragazzi di origine immigrata, quasi tutti iscritti ad un istituto professionale - precludendosi, in parte, la possibilità di una formazione universitaria - e spesso costretti ad abbandonare il loro percorso scolastico per fare ingresso nel mercato lavorativo a causa delle necessità materiali delle famiglie colpite dalla crisi più spesso e più pesantemente rispetto alle famiglie autoctone (Bonifazi and Marini 2014; Fondazione Leone Moressa 2015).

Mio papà aveva perso il lavoro. Mio padre lavorava da solo e non prendeva tanto, mio fratello, siamo quattro persone, io e mio fratello, quindi ho detto anch'io aiuto te, do una mano alla mia famiglia. (Ratan, 20 anni, origine immigrata, lavoratore, residente a Mestre)

A ciò si aggiungono poi le difficoltà linguistiche della famiglia di origine e, quindi, il minore sostegno familiare nei processi di apprendimento scolastico, ma anche la struttura delle politiche migratorie in Italia e alcune dinamiche proprie della scuola italiana. Da un lato, la scuola orienta gli alunni stranieri soprattutto verso percorsi di formazione breve in virtù di loro supposte "inclinazioni - o distanze - culturali" o della loro condizione di difficoltà, dando vita ad una vera e propria "profezia autoadempiente". Dall'altro lato le politiche relative all'ingresso e alla residenza degli immigrati, legando la regolarità del soggiorno al possesso di un contratto di lavoro, inducono gli alunni stranieri - spesso su spinta delle loro famiglie - a optare per un inserimento in quegli istituti che si presume possano garantire un più rapido ingresso nel mercato del lavoro, anche se nei segmenti più bassi e meno retribuiti, o addirittura per un abbandono precoce in vista di un qualsiasi inserimento lavorativo purché in tempi brevi. La scelta di percorsi formativi a breve termine, inoltre, sarebbe influenzata dalle possibilità economiche della famiglia: la decisione di frequentare un liceo è subordinata alla possibilità di sostenere economicamente un percorso di studi che proseguirà con l'istruzione universitaria e, quindi, più lungo (Dei 2000; Queirolo Palmas 2006).

Molti hanno problemi in famiglia, molti vorrebbero vedere i figli studiare in una scuola tecnica e non in una scuola normale come me, così dopo tre anni puoi andare subito a lavorare o molti li mandano a scuola solo perché c'è l'obbligo fino ai 16 anni e appena li compi scappano dalla scuola e vanno a lavorare. I genitori non capiscono che se magari adesso lavorerà come vogliono, ma in futuro se li facevano stu- 
diare avrebbero avuto qualche carta in più da giocare nel mondo del lavoro. (Ali, 17 anni, origine immigrata, studente, residente a Mestre)

Relativamente ai giovani di origine immigrata residenti a Mestre e Marghera, quindi, non è tanto il quartiere di residenza a incidere negativamente sulle loro traiettorie scolastiche (Sykes e Kuyper 2009), quanto la condizione di svantaggio strutturale data dalla loro origine immigrata e, di conseguenza, dalla collocazione di classe delle loro famiglie in Italia.

\subsection{Il genere: ambivalenze e contraddizioni}

Per quanto riguarda l'appartenenza di genere, tutti gli intervistati, di entrambi i quartieri, concordano nell'affermare che per le ragazze risulta più facile trovare un'occupazione lavorativa. Aggiungono anche, però, che ciò avverrebbe solo ed esclusivamente in specifici segmenti del mercato del lavoro come quello turistico, della ristorazione, nei bar e nei locali pubblici e, più in generale, nel terziario a bassa qualifica:

Per trovare lavoro forse [le ragazze] hanno anche qualche vantaggio. Anche se è paradossale. Considera che Venezia, qua a Marghera e dintorni, la maggior parte delle attività sono... bar! Nei bar vogliono le ragazze. Oppure molte lavorano a Venezia, sempre nel turismo. (Maurizio, 23 anni, origine autoctona, studente e lavoratore, residente a Marghera)

Per una ragazza qua a Mestre è molto più semplice trovare un lavoretto. $\mathrm{OK}$, ma ovviamente non è il lavoro della vita. Sono avvantaggiate nella facilità di trovare un lavoro per quanto riguarda lavoretti piccoli: barista, banconiera, cameriera... Ovviamente tutti capiscono il vantaggio di avere una bella presenza e, quindi... Però avere anche questi paletti qua per lavorare è brutto, veramente brutto. (Mirko, 22 anni, origine autoctona, studente, residente a Mestre)

L'unica cosa secondo me, a Mestre i posti di lavoro che sono rimasti sono più accessibili per una ragazza...a Mestre. Perché cosa va? La barista, la commessa. Perché cosa guardano tanto? La bella presenza, capito? Perciò, per un ragazzo trovare lavoro, a Mestre magari è più difficile. Una ragazza fa un giro per qualche negozio, in centro, e trova qualcosa. Mentre magari un ragazzo ha più difficoltà perché è sempre richiesta la bella presenza femminile... (Mirko, 22 anni, origine autoctona, studente, residente a Mestre).

Ciò va ricondotto allo stereotipo che giustifica la selezione di "ragazze di bella presenza" per specifici inserimenti lavorativi a contatto col pubblico e che, se da un lato, sembrerebbe avvantaggiare la componente femminile della forza-lavoro più giovane, dall'altro lato, confinerebbe le ragazze in determinati settori lavorativi a svolgere le mansioni più semplici e meno retribuite. Soprattutto, però, va connesso alla struttura economica e del mercato del lavoro della Città di Venezia e alla sua 
"vocazione turistica": il pressoché totale sbilanciamento - economico, politico e produttivo - verso il settore turistico che caratterizza la Città e, soprattutto, il Centro Storico ${ }^{4}$ non può non influenzare anche le traiettorie lavorative, identitarie e sociali dei quartieri della Terraferma. Le stesse narrazioni degli intervistati, infatti, descrivono il progressivo restringimento delle possibilità di inserimento lavorativo che non siano quelle di bassa manovalanza nel settore turistico, ristorativo, alberghiero e la differenziazione delle traiettorie lavorative di ragazzi e ragazze. Nonostante 1'“ipertrofia" del settore turistico veneziano $\mathrm{o}$, più probabilmente, proprio per questa ragione, va sottolineato che la Città di Venezia è caratterizzata da un tasso di disoccupazione del 25\% (Bresolin 2017). Stando alle parole degli intervistati, inoltre, le ragazze sarebbero meno esposte a fenomeni di devianza e attività illegali - che, nel contesto di Mestre e Marghera, come è stato anticipato, si tradurrebbe nella vendita e nel consumo di sostanze stupefacenti. Gli stili, gli spazi e i tempi della socialità socialmente costruiti come "maschili" e "femminili", le differenti aspettative familiari e sociali riversate su ragazzi e ragazze (Goffman 1977), il maggior controllo esercitato dalla famiglia dissuaderebbero le ragazze dal partecipare assiduamente alle attività di aggregazione informale praticata, negli spazi pubblici (parchetti, piazze, panchine...), soprattutto dalla componente maschile del gruppo dei pari - entrando, così, più difficilmente in contatto diretto con l'offerta di sostanze stupefacenti:

Forse le ragazze stanno un po' più attente o sono più sotto controllo dai genitori. [...] In un quartiere come Marghera, mi sembra più facile che nella tossicodipendenza ci caschi più un ragazzo, anche perché vive di più la realtà di stare fuori, la strada. Una ragazza sta fuori meno, nel senso... un ragazzo riesce a star fuori fino alle sette, una ragazza torna a casa prima. Forse dal punto di vista interiore, da come la vive una ragazza, il fatto di essere in un quartiere considerato pericoloso... andare in giro per strada a certe ore... ha paura. L'ho già sentito da ragazze della mia età. Se, invece, sei un ragazzo... sei pur sempre un ragazzo di Marghera e devi essere un po' un bulletto, no? [...] Mentre per le ragazza è meno pericoloso - a parere mio -, ma se la vivono male, se la vivono peggio, meno pericolo, ma allo stesso tempo ti mette più insicurezze diciamo così. (Maurizio, 23 anni, origine autoctona, studente e lavoratore, residente a Marghera)

Come emerge dalle narrazioni degli intervistati, quindi, è la stessa interiorizzazione della paura di transitare in aree del quartiere rappresentate come "pericolose" che spingerebbe le ragazze a preservarsi maggiormente rispetto ai coetanei di gene-

${ }^{4} \mathrm{Nel} 2015$, la Città di Venezia ha contato 4,5 milioni di arrivi (numero fisico di persone non residenti che arrivano nel territorio e vi trascorrono almeno una notte) e oltre 10 milioni di presenze (il numero complessivo dei pernottamenti). A questi dati vanno aggiunti i 15 milioni di turisti che non pernottano, ma vi trascorrono la giornata dalla mattina alla sera e i 3,6 milioni che visitano Venezia pernottando fuori Comune (Città di Venezia 2015). Altre fonti parlano genericamente di 30 milioni di visitatori l'anno (Bresolin 2017). In qualsiasi caso, queste cifre e il corrispettivo fatturato rendono possibile parlare di una vera e propria "monocultura turistica" veneziana. 
re maschile. Le interviste, dunque, palesano disuguali possibilità di fare esperienza degli spazi e dei tempi urbani sulla base della dimensione di genere (Fenster 2005). Le ragazze, cioè, si sentirebbero meno libere di transitare in specifiche porzioni di quartiere, di utilizzare determinati spazi pubblici di vivere il tessuto urbano in alcuni momenti della giornata:

C'è sempre il concetto che la ragazza deve avere sempre più paura, è vero che la ragazza per sua auto protezione deve avere un occhio in più, [...] in ogni caso è meglio sempre avere un occhio dietro la testa. Questa paura è instillata anche dall'esterno e proprio dall'ambiente e dalle persone di Mestre, quindi se sei ragazza non puoi andare fuori da sola, devi stare sempre attenta, devi muoverti con qualcuno... Diciamo che il maschio è il primo a fare gruppo, il primo che esce anche in determinate zone, mentre alcune di noi ragazze, come abbiamo detto prima, hanno paura a muoversi per Marghera di sera da sole (Enrica, 20 anni, origine autoctona, studentessa, residente a Mestre)

Marta: Io, ragazza da sola, non posso farmi da sola via Piave: rischio la vita. Anche ad andare in stazione a Mestre, anche di giorno, ho paura. O per andare a prendere il treno arrivo due minuti prima, non sai mai cosa ti può succedere!

Sandra: In settimana io non sono mai in giro dopo le otto. Il fine settimana, ovunque io vada, ovunque io sia, ho sempre il coltellino dietro... anche se dovesse fermarmi la polizia e mi dicono che è illegale, non mi interessa, io personalmente non mi fido di nessuno.

Marta: Quando la sera tornavo da Venezia io giravo con le chiavi in mano che se qualcuno mi avvicinava gliele lanciavo addosso, così si perde via, si distrae un attimo e puoi scappare. Me l'ha insegnato mia nonna. Se incontri uno sbandato che cazzo fai? Se chiamo i carabinieri chissà quando arrivano? [...] Noi donne ci sentiamo più fragili. Arrivi a pensare a queste cose. Quando sono in stazione a Mestre spero di arrivare a casa. (Focus group)

Una mia amica stava tornando a casa a Marghera, in un quartiere abbastanza residenziale, e un tipo l'ha cominciata a seguire chiedendogli prestazioni sessuali a pagamento per dirne una... A Marghera è una cosa quasi di routine che fermano le ragazze chiedono se sono prostitute... sono cose orribili. (Mirko, 22 anni, origine autoctona, studente, residente a Mestre).

Le attese sociali e il controllo familiare - e "socio-comunitario" -, che strutturerebbero in maniera diseguale le traiettorie lavorative, sociali, relazionali e della mobilità urbana di ragazzi e ragazze, sembrerebbero amplificarsi e agire con maggior "efficacia" all'interno della collettività bangladese - la più numerosa tra le collettività di origine "extra-comunitaria" -, così come espresso dagli intervistati (Clark et al. 2017). La componente femminile dei giovani di origine bangladese residenti nei quartieri, infatti, disporrebbe di minor autonomia decisionale e di movimento e, di conseguenza, sarebbe meno esposta al coinvolgimento in attività devianti: 
Essere maschio, per la mentalità bengalese, può andare da ogni parte, girare... Una donna ha molti limiti, una ragazza non può andare in giro con quel tipo di vestiti, parlano dietro, lo dicono ai genitori, hanno dei limiti, non possono andare in posti particolare tranne i supermercati... Per le famiglie italiane non lo so, ma per quelli bengalesi ho sperimentato. (Ali, 17 anni, origine immigrata, studente, residente a Mestre)

Secondo me più i ragazzi rischiano di più di entrare in "brutti giri" delle ragazze, soprattutto perchè a questa età sono i ragazzi che fanno uso di droghe, che escono sempre... [...] Mio fratello ha 25 anni e certe volte torna a casa tardissimo di notte, se io avessi 25 anni e tornassi tardi magari alle 5 del mattino mia mamma non mi lascerebbe più uscire, quindi non è giusta questa cosa, se questa cosa vale per me dovrebbe valere anche per lui. Nella comunità bengalese c'è una grossa differenza fra le femmine e i maschi e questa è una cosa che mi turba moltissimo, è una delle cose che vorrei cambiare in futuro. [...] Ovviamente non penso che i genitori italiani lascino uscire la figlia di 16 anni tutti i giorni, che tornino a casa a mezzanotte e mezza, però, ad esempio, le lasciano fare sport, le lasciano uscire non solo con le amiche ma anche con gli amici e di sabato possono tornare tardi, andare in discoteca oppure ai compleanni anche fino a mezzanotte e queste cose io non possono permettermele... (Rokeya, 16 anni, origine immigrata, studentessa, residente a Marghera)

Essere una ragazza bengalese qua è difficile. Le ragazze non lavorano da noi, adesso è un po' cambiato. Per una ragazza lavorare qua, sarà difficile. Se vedono lavorare una ragazza parlano alle spalle...parlano alle spalle in maniera negativa. Nel lavoro, se una ragazza finisce a mezzanotte, se è italiana $\mathrm{OK}$ se è bengalese non va bene... Poi non possono uscire, stare fuori oltre le nove di sera. Non possono stare dopo le nove. (Pryon, 22 anni, origine immigrata, studente lavoratore, residente a Marghera)

In generale, però, tutti gli intervistati di origine bangladese, accusano il peso di una soffocante sorveglianza "comunitaria": un dispositivo di controllo "panottico" costituito dallo sguardo, dall'ascolto e dalla voce degli adulti appartenenti a quella che, talvolta impropriamente, viene definita la "comunità dei connazionali" che sorveglia e condiziona le condotte, le frequentazioni, gli stili della socialità, le estetiche dei giovani e che riferisce alle loro famiglie di origine rispetto a ciò:

Ho meno possibilità per il fatto che ci sono tanti miei connazionali qua, tanti bengalesi, non lo vedo come un fastidio in sé, ma comunque non è il massimo diciamo. $\mathrm{Nel}$ senso che qualsiasi cosa che fai attiri molta attenzione dei tuoi connazionali che magari parlano, non so come succede... se andassi in giro a Marghera con una ragazza sarebbe un problema, è una piccola comunità e la gente sparlerebbe. Soprattutto per i miei connazionali. Parlano tra di loro e la comunità è piccola e quindi tutti vengono a sapere. La gente mi conosce, sanno chi sono. Non lo so come fanno, però sanno chi sono, cosa faccio e cosa no. (Maliq, 21 anni, origine immigrata, lavoratore, residente a Marghera) 
Una cosa negativa è che parlano tra di loro, non si fanno mai gli affari loro, dicono cose che non sono assolutamente vere e quindi circola tutto. (Rokeya, 16 anni, origine immigrata, studentessa, residente a Marghera)

Adesso sono sotto pressione, ogni parte che vado, ogni scelta che faccio mi sento sotto osservazione... da gente sconosciuta che, però, magari conosce i miei genitori. (Ali, 17 anni, origine immigrata, studente, residente a Mestre)

\subsection{La dimensione "etnico-nazionale”: discriminazione e marginalizzazione}

Oltre all'onnipresente controllo della "comunità" dei connazionali (Bertani e Di Nicola 2012) - che i giovani bangladesi percepirebbero come una forma di diseguaglianza rispetto ai coetanei di origine autoctona - e alla già descritta disparità rispetto alle opportunità scolastico-formative, i ragazzi di origine immigrata sconterebbero condizioni di diseguaglianza nell'ambito lavorativo e sarebbero vittime di discriminazioni:

C'è abbastanza razzismo [...] se, però, sei immigrato e vieni qua, la maggior parte della gente ti guarda storto perché ci togli il lavoro, per tanti, soprattutto i datori di lavoro, questi sono un'ottima opportunità perché spesso succede che il datore di lavoro assume queste persone immigrate che vengono pagate meno, perché magari assunte in nero o perché semplicemente vengono pagate meno perché va bene così e questi, magari, non dicono niente, lavorano anche venti ore al giorno. [...] Lo vedi proprio, io avevo un compagno alle medie nigeriano che spesso lo si scherzava sul fatto che era di colore. Vedo che è una cosa di mentalità radicata nelle persone, non è una cosa che si fa intenzionalmente, tante persone hanno questo lato razzista che esce fuori non si sa bene perché...c'è chi lo tira fuori in maniera aggressiva e chi fa la battuta così che va beh, cioè...però ci sono state anche persone che conosco, amici che sono andati in un posto di lavoro e hanno detto: "Tu di dove sei? - Sono moldavo - Ah sei moldavo, non ti assumo perché cerco solo un ragazzo italiano! - Avevi detto che cercavi uno che parlava le lingue e io parlo le lingue! - Eh si però preferisco un ragazzo italiano". (Alvise, 21 anni, origine autoctona, studente lavoratore, residente a Marghera)

Purtroppo mi rendo conto che viviamo in un posto, in un tempo, in un luogo in cui uno pensa allo straniero, all'immigrato come manchevole di qualche cosa. Tante volte $\mathrm{i}$ bambini stranieri, io faccio l'esempio della scuola, si pensa siano un po' più stupidi perché non conoscono la lingua ma non ci si rende conto che ne conoscono un'altra. (Chiara, 21 anni, origine autoctona, studente lavoratrice, residente a Mestre)

A me da fastidio che quando vado a comprarmi qualcosa e chiedo alla commessa di aiutarmi, perché non capisco qualcosa su un prodotto, quella continua magari a parlare con una signora italiana e non mi guarda perché sono straniera e non è piacevole. (Rokeya, 16 anni, origine immigrata, studentessa, residente a Marghera) 
Generalmente, ciò emerge dalle narrazioni dei giovani autoctoni, ma non da quelle dei diretti interessati che, al contrario, negano qualsiasi forma di discriminazione "etnico-razziale" o diseguaglianza basata sull'origine nazionale. Tale diseguaglianza trapela comunque dalla voce degli intervistati di origine immigrata nonostante, nelle costruzioni discorsive, venga negata. Ciò può essere spiegato con il senso di vergogna provato di fronte all'intervistatore per l'eventuale ammissione della propria condizione di subalternità socialmente costruita, con la negazione - in primo luogo a sé stessi - dell'umiliazione di essere o essere stati protagonisti di episodi di discriminazione, con la volontà di ostentare uno scarto fra le esperienze della generazione dei propri genitori primomigranti, segnata dalla disillusione dell'immigrazione (Sayad 1999), dal confinamento in specifici settori lavorativi (Ambrosini 2005; Perocco 2012; Zanfrini 2010) - i così detti "lavori delle 5 P" (Ambrosini 2005) - con il perseguito smarcamento, cioè della figura del "manovale a vita" (Sayad 1999) a cui si sono adeguati i loro padri.

Non è che ce l'ho col mio Paese, ma quelli che adesso sono arrivati e sono bengalesi, io li odio, quella cosa che mi dà fastidio: i datori di lavoro li trattano male perché non capiscono l'italiano, quindi lavorano tanto e prendono poco. Se io vado là con il mio $\mathrm{CV}$ e vado là a chiedere lavoro mi dicono subito di no, perché sa già che io non voglio accettare tutto, capisco l'italiano, so i miei diritti, ho i documenti, quindi, per colpa loro, io non riesco a trovare lavoro. Sono arrabbiato per questa cosa. Se io vado là e se dice qualcosa sbagliata io gli rispondo, non può ingannarmi, se mi fa lavorare di più mi deve dare più soldi. Invece a loro li trattano male, ti fanno lavorare di più, danno pochi soldi e loro zitti. (Pryon, 22 anni, origine immigrata, studente lavoratore, residente a Marghera)

\subsection{La dimensione intergenerazionale: eternamente "in formazione”}

Infine, le parole di alcuni intervistati hanno messo in luce delle diseguaglianze riprodotte rispetto alla direttrice generazionale: il fatto di essere giovani di per sé sarebbe motivo di esclusione dalla sfera politica e lavorativa e di screditamento professionale. Tale aspetto risulta evidente dalle parole di Elisa, 23 anni e ostetrica da oltre due anni, la cui giovane età se da un lato è motivo di discredito e scarsa fiducia nelle sue competenze, dall'altro è usata come pretesto per abbattere il costo del suo lavoro e della sua professionalità:

Ho in mano competenze, anche molto pratiche, e poi, però, mi scontro sempre con un mondo che vede il giovane come un bambino. Quindi tu, anche se hai in mano un titolo, non ti considerando. [...] Ho fatto un sacco di corsi di aggiornamento, ho speso più di quello che guadagnavo, grazie anche ai miei genitori. Insomma, continuo a continuare a formarmi e anche adesso sto facendo un master privato molto costoso. Poi, però - io lavoro anche con donne in menopausa per dire - se mi trovano già in ambulatorio loro mi vedono come un'altra figura che non va. OK, ho il camice addosso e, quando parli loro, riescono a capire la tua professionalità. Però appena uno sente "giovane" ti chiedono: "Giovane quanto?". Io ho 23 anni, ma non dico mai la 
mia età dico: "Eh giovane, me lo dicono tutti", perché mi rendo conto che è difficile per una persona più vecchia prenderti in considerazione come una professionista. Questa mentalità che uno è giovane è una bambina e non potrà mai essere al pari di una che ha 40 anni. OK, magari non ho certe esperienze, però, in realtà per certe cose, insomma, io vedo che persone anche più vecchie, nel mio campo, passano alle mamme magari informazioni sbagliate, che derivano da pensieri un po' antichi se vuoi. Però questo non sembra importante come l'età: loro guardano se sei vecchia o meno. Nei posti dove ti presenti, ti calcolano un cavolo, ti dicono: "Sì mi vieni a fare questa cosa, ti pago in nero, ti pago poco". Vieni trattato come una bambina. (Elisa, 23 anni, origine autoctona, lavoratrice, residente a Mestre)

Ancora Elisa, nell'interazione con un coetaneo:

Elisa: Siamo giovani, quindi se tu ti vuoi affacciare come libero professionista ti guardano sempre dall'altro al basso, perché i ginecologi sono vecchi per la maggior parte quindi ti guardano dall'alto della loro età, noi ci laureiamo giovani, però ciò non vuol dire che non siamo capaci. Tutto per questo scarto generazionale, perché sei giovane, perché l'immagine dell'ostetrica esperta è quella che ha fatto un sacco di anni in ospedale il problema è che adesso è impossibile entrare in ospedale! Quindi adesso bisogna inventarsi qualcosa e io ci sto provando, sembra che funzioni... Quindi la mancanza di fiducia arriva dalle istituzioni, $[. .$.$] i medici non ti cal-$ colano, ma io ho una laurea in mano, ho della pratica in mano!

Fabio: Accade la stessa per noi, per gli assistenti sociali! È uguale, identico. (Focus group)

Ma emerge anche in quelle di Mirko, 22 anni, organizzatore di molti eventi pubblici di successo all'interno del suo quartiere, ma - a suo dire - poco ascoltato, se non proprio boicottato, dalle istituzioni della municipalità a causa della sua giovane età:

Ti tolgono tutte le possibilità tu puoi anche avere l'idea più bella del mondo a zero soldi per il Comune o la Municipalità, un'iniziativa che magari porta soldi, porta turismo eccetera, ed è automaticamente bocciata perché te che la vai a proporre sei un ragazzo, quindi, non hai esperienza sei uno sbarbato, chissà chi sei... (Mirko, 22 anni, origine autoctona, studente, residente a Mestre)

\section{Conclusioni}

La raccolta di interviste narrative con i giovani tra i 16 e i 25 anni, residenti nei quartieri di Mestre e Marghera, della Città di Venezia, ha permesso di osservare come essi facciano un'esperienza pluridimensionale e intersezionale delle disuguaglianze e come la residenza e la vita quotidiana in un determinato quartiere, da un lato, generi schemi percettivi, perimetrazioni, analisi delle forme di appartenenza, identità e radicamento locale e, dall'altro lato, possa riprodurre traiettorie di esclu- 
sione connesse a dinamiche individuali e strutturali di deprivazione e a specifici effetti del contesto spaziale stesso (Borlini 2010; Wilson 1987).

$\mathrm{Al}$ contempo, è emersa l'importanza della specificità scalare del contesto "locale" e della sua storia nel declinare e strutturare l'esperienza delle diseguaglianze.

Va innanzitutto tenuta in conto l'eredità storica nella produzione e riproduzione delle diseguaglianze nello specifico cotesto di Mestre e Marghera. I due quartieri, infatti, presentano una storia che li ha profondamente segnati, legata in parte a scelte politiche originarie a partire dal primo dopoguerra fino agli anni Cinquanta e in parte al cambiamento dello scenario economico e produttivo. La vicinanza e l'annessione alla Venezia capitale della cultura e del turismo hanno condizionato la loro evoluzione, seppur con differenze importanti tra i due contesti che - proprio come conseguenza di questa evoluzione storica - condividono una relazione di separazione geografica e, soprattutto, sociale. Di per sé, infatti, già la posizione di un quartiere nel contesto urbano gioca un ruolo importante nell'esperienza di inclusione o esclusione, ma anche nel contribuire alla produzione di uno stigma negativo che colpisce i residenti. Come è stato mostrato, tale "separatezza" è il risultato di un'effettiva e originariamente intenzionale separazione attuata dalle politiche locali del passato. A Marghera, infatti, alcune specifiche porzioni del quartiere sono state esplicitamente progettate per un'informale confinamento dei soggetti "unwanted", un fenomeno ben analizzato da Ash Amin che lo ha definito "telescopic urbanism" e lo ha descritto nei termini di un "sanguine and disengaged approach to the urban poor [...] in which only particular parts of the city are projected as spaces of potentiality or attention" (Amin 2013, 477). Vanno prese in considerazione anche distanze di natura simbolica che producono quelle che la tradizione sociologica ha già definito "città nella città" (Park and Burgess, 1928) o che possono essere individuate come "inner peripheries" (ESPON 2012, Bromley 1997). Gli elementi che, nella mappa mentale dei residenti, separano simbolicamente, prima ancora che spazialmente, i quartieri sono costituiti dalla ferrovia e dalla stazione di Venezia Mestre, in primis, ma anche dalla Strada Statale 11 e dal Ponte della libertà. Servizi, scuole, campi da gioco, luoghi di aggregazione e spazi di leisure potrebbero essere raggiunti in pochi minuti, ma non vengono utilizzati perché sono pensati e narrati come luoghi (per) altri e/o pericolosi (Güentner et al. 2018; Campomori e Della Puppa 2014; 2017). Politiche locali efficaci dovrebbero, quindi, tenere conto delle mappe mentali dei residenti, ancor prima che di quelle della pianificazione urbana e architettonica (Pezzi e Punziano 2017). La relazione tra le direttrici lungo le quali si struttura l'esperienza della diseguaglianza e la storia dei contesti urbani e delle scelte politiche locali si palesa anche relativamente alla dimensione strettamente economica. È significativo, infatti, che questi territori siano stati particolarmente segnati dalla crisi industriale e dal tracollo dell'occupazione che hanno contribuito a stratificare la composizione sociale dei residenti fra cui si annoverano famiglie di ceto impiegatizio, famiglie "tradizionalmente" operaie e famiglie immigrate i cui principali breadwinner trovano occupazione, oltre che nel settore turistico, alberghiero e ristorativo, nel sistema delle cooperative in subappalto che orbitano attorno al ridimensionato distretto industriale. 
Il processo di deindustrializzazione, reso ancora più intenso dall'impatto della crisi economica, unitamente a quella che è stata definita l'ipertrofia del settore turistico nel contesto veneziano, parrebbero ricoprire un ruolo nella strutturazione dell'esperienza di diseguaglianza lungo direttrici di genere. Rispetto a tale dimensione, infatti, il fieldwork ha restituito risultati apparentemente inediti e parzialmente inaspettati, poiché emergerebbe una condizione di apparente "privilegio" a vantaggio della componente femminile nel mercato del lavoro locale, proprio in virtù delle specifiche dinamiche economiche locali che ancorerebbero saldamente le sorti economiche del contesto urbano al comparto turistico (Iannuzzi 2017). Al contempo, però, il facile inserimento della giovane forza-lavoro femminile, rispetto a quella maschile, nel settore alberghiero, ristorativo e del commercio comporterebbe anche concreti rischi di segregazione occupazionale nel terziario a bassa qualifica (Cavaletto e Olagnero 2015; Poggio 2006; Rosti 2006). La dimensione di genere, inoltre, strutturerebbe anche l'esperienza urbana della componente più giovane dei residenti. Le costruzioni normative relative "all'essere un ragazzo" o "essere una ragazza", infatti, modellano gli habitus dei soggetti e, con essi, la frequentazione di determinati spazi e tempi del contesto di residenza (Fenster 2005).

Tale dinamica parrebbe ancor più concreta per i giovani di origine immigrata. Questi ultimi, inoltre, da un lato, attraverserebbero una quotidianità costellata da episodi di discriminazione negli spazi urbani e negli esercizi pubblici, nella scuola e nel mercato del lavoro locale, ambiti in cui sono ripetutamente costruiti come "stranieri" a causa, soprattutto, dei loro caratteri somatici; dall'altro lato, accuserebbero il peso del controllo da parte dei membri della collettività dei "connazionali" che, a loro volta, li riconduce a dinamiche di appartenenza "etnica". Soprattutto, gli intervistati di origine immigrata, esprimono il rifiuto per la condizione di subalternità sociale e lavorativa dei loro padri primomigranti e alla canalizzazione verso il già citato terziario a bassa qualifica che caratterizza l'economia veneziana, aspirando a una mobilità ascendente rispetto alla collocazione sociale che la migrazione avrebbe assegnato alle loro famiglie in Italia (Della Puppa 2014). La società e il mercato del lavoro italiani, cioè, li percepirebbero e rappresenterebbero come forza-lavoro destinata, sin da subito, ai così detti "lavori delle $5 \mathrm{P}$ " (precari, pesanti, pericolosi, poco pagati, penalizzanti socialmente) (Ambrosini 2005), diversamente da quanto avviene per i giovani di origine autoctona, costruiti socialmente come "permanentemente giovani" ed "eternamente in formazione" in quanto la loro collocazione generazionale sarebbe motivo di esclusione dalla sfera politica e culturale, lavorativa e professionale. Tale aspetto, emerso dalla narrazione delle esperienze da parte degli intervistati appare ancora più paradossale tenendo in considerazione il tasso strutturale relativo alla disoccupazione giovanile per la fascia di età in considerazione (che sfiora i 40 punti percentuale) ${ }^{5}$. Soprattutto, come mostrato in letteratura (Caltabiano and Rosina 2018), simili svantaggi generazionali non

5 Anche se, nel leggere tale dato, va tenuta in considerazione la lunga durata della formazione scolastica dei giovani in Italia. 
solo ostacolano la crescita economica a livello locale e nazionale ma, in una prospettiva intergenerazionale e familiare, esacerbano anche le disuguaglianze sociali.

Se lo svantaggio dei giovani di origine immigrata e dei giovani in quanto tali non costituiscono dei tratti distintivi dei quartieri di Mestre e Marghera - anche se in tali contesti si declinano secondo specificità locali -, certamente le modalità attraverso le quali prendono forma le diseguaglianze lungo direttrici spaziali, sociali, "etnico-razziali" e di genere assumono aspetti peculiari nei territori analizzati, a causa del contesto territoriale, economico e culturale entro i quali sono inseriti. Saper districare l'intreccio di tali variabili per meglio ascoltare e comprendere la voce degli “inascoltati” è una sfida dalla quale gli attori locali non possono esimersi.

\section{RIFERIMENTI BIBLIOGRAFICI}

Agustoni, A. e Alietti, A. 2009. Società urbane e convivenza interetnica. Milano: FrancoAngeli.

Ambrosini, M. 2005. Sociologia delle migrazioni. Bologna: Il Mulino.

Amin, A. 2013. Telescoping Urbanism and the Poor, City, 17(4), pp. 476-492.

Anthias, F. 2008. Thinking through the lens of translocational positionality: an intersectionality frame for understanding identity and belonging'. Translocations, Migration and Change, 4(1), pp. 5-20.

Atkinson, R. e Kintrea, K. 2004. 'Opportunities and despair, it's all in there': practitioner experiences and explanations of area effects and life chances, Sociology, 38(3), pp. 437-55

Azzolini, D., Poy, S e Schizzerotto, A. 2013. Giovani, Mondo del lavoro, condizioni di vita e politiche pubbliche. In Osservatorio del mondo del Lavoro (a cura di), XXVIII Rapporto sull'occupazione in provincia di Trento - 2013, pp. 181-207. Milano: FrancoAngeli.

Ballarino, G. e Checchi, D. 2006. Sistema scolastico e disuguaglianza sociale. Scelte individuali e vincoli strutturali. Bologna: Il Mulino.

Barizza S. e Cesco L. a cura di. 2007. Marghera 1917-2007. Voci, suoni e luci tra case e fabbriche. Venezia: Centro Francescano di cultura.

Barizza S. a cura di. 2000. Marghera. Il quartiere urbano. Alcione: Venezia.

Barbieri, P. e Fullin, G. (a cura di). 2014. Lavoro, istituzioni, diseguaglianze. Bologna: Il Mulino.

Bello, B.G. and Cuzzocrea, V. 2018. Introducing the need to study young people in contemporary Italy, Journal of Modern Italian Studies, 23(1), pp. 1-7.

Bertani, M. e Di Nicola, P. 2012. Presentazione, Sociologia e politiche sociali, 1, pp. 5-7.

Bonifazi, C. and Marini. C. 2014. The Impact of the Economic Crisis on Foreigners in the Italian Labour Market. Journal of Ethnic and Migration Studies, 3(40), pp. 493-511.

Borlini, B. 2010. Il quartiere nella città contemporanea. Come e perché occuparsene. Quaderni di Sociologia, 52, pp. 13-29. 
Bourdieu , P. 1979. Symbolic Power. Critique of Anthropology, 4, pp. 77-85.

- 1993. Effets de lieu. In P. Bourdieu ed. La Misère du monde, pp. 249-250. Paris: Seuil.

Bresolin, A. 2017. Venezia: Albergo diffuso galleggiante. Lo Squaderno. Explorations in Space and Society, 45, pp. 31-33.

Bricocoli, M. e Padovani, L. 2006. La fatica di superare la città a due velocità. Uno sguardo retrospettivo su 25 anni di politiche di quartiere in Francia. Animazione sociale, XXXVI(208).

Brighenti, A.M., Mattiucci, C. and Rahola, F. eds 2016. Special Issue: Segni di confine | Urban bondaires. Urban Tracks, VIII(21).

Bromley, R. 1997. Globalization and the inner perifery: A Mid Bronx View. Annals of the American Accademy of Political and Social Sciences, 555, pp. 191207.

Caltabiano, M. and Rosina, A. 2018. The dejuvenation of the Italian population, Journal of Modern Italian Studies, 23(1), pp. 24-40.

Campomori, F. e Della Puppa, F. 2014. Innovazione sociale ed empowerment dei cittadini: il caso dell'Etam di Venezia. Rivista di servizio sociale, 54(2), pp. $17-38$.

- 2017. Venice. In Guentner, S., Seukwa, L.H., Gherke, A.M. and J. Robinson (eds). Local matters? Neighbourhoods and social infrastructure as spaces of reproducing, producing, mitigating or counteracting social inequalities in 10 European cities, Hamburg: peter Lang.

Carrosio, G. 2016. A place-based perspective for welfare recalibration in the Italian inner peripheries: the case of the Italian strategy for inner areas. Sociologia e politiche sociali, 3, pp. 50-64.

Casarin M. e Saccà G. 2009. Mestre, città nuova. Marcianum Press: Venezia.

Cavaletto, G.M. e Olagnero, M. 2015. Lavoro è potere? SegregazionE occupazionale e leadership femminile, Giurisprudenza italiana, dicembre, pp. 2248-2256.

Città di Venezia. 2015. Annuario del turismo 2015. Venezia: Assessorato al Turismo.

Clark, A.V., Duque-Cavache R. e Palomare-Linares, I. 2017. Place Attachment and the Decision to Stay in the Neighbourhood. Population, Space and Place. Doi: $10.1002 /$ psp. 2001

Colombo, E. and Rebughini, P. 2016. Introduction, Rassegna italiana di sociologia, (LVII)3, pp. 415-420.

Colombo E., Leonini, L. and Rebughini, P. 2018. A generationa attitude: young adults facing the economic crisis in Milan, Journal of Modern Italian Studies, 23(1), pp. 61-74.

Crenshaw, K.W. 1989. Demarginalizing the intersection of race and sex: a black feminist critique of antidiscrimination doctrine, feminist theory, and antiracist politics. University of Chicago Legal Forum, 1, pp.138-167. 
1991. Mapping the Margins. Intersectionality, Identity Politics, and Violence against Women of Colour, Stanford Law Review, 43(6), pp. 1241-1299.

Davis, K. 2008. Intersectionality as buzzword: A sociology of science perspective on what makes a feminist theory successful. Feminist Theory, 9(1), pp. 67-85.

Della Puppa, F. 2014. Uomini in movimento. Il lavoro della maschilità tra Bangladesh e Italia. Torino,: Rosenberg \& Sellier.

Della Puppa, F. e Gelati, E. 2015. Alte Ceccato. Una banglatown nel nordest. Trento: Professionaldreamers.

Dei, M. 2000. La scuola in Italia. Bologna: Il Mulino.

Denton, N. and Massey, D. 1993. American Apartheid: Segregation and the Making of the Underclass. Harvard: Harvard University Press.

ESPON 2012. European Perspective on Specific Types of Territories. Final Report. Geneva: University of Geneva.

Farrugia, D., 2015, "Space and Place in Studies of Childhood and Youth" in Wyn J., Cahill H. (eds.), Handbook of Children and Youth Studies, Springer: Singapore, pp. 609-624

Fenster, T. 2005. The Right to Gendered City. Different Formations of Belonging in Everyday Life. Journal of Gender Studies, 14(3), pp. 217-231.

Fondazione Leone Moressa. 2015. Rapporto annuale sull'economia dell'immigrazione. Edizione 2015. Bologna: Il Mulino.

Gabbi, F. 2013. "Vicino Ma Fuori”. Il quartiere di San Martino a Trento. Trento: Professionaldreamers.

Gallino, L. 2000. Globalizzazione e diseguaglianze. Roma-Bari: Laterza.

Güentner, S., Seukwa, L.H., Gherke, A.M. and J. Robinson (eds). Local matters? Neighbourhoods and social infrastructure as spaces of reproducing, producing, mitigating or counteracting social inequalities in European cities, Hamburg: Peter Lang.

Goffman, E. 1977. The Arrangement between the Sexes. Theory and Society, 4, pp. 301-331.

Gosetti, G. 2007. Lavori, disuguaglianze e protezione sociale. Milano: FrancoAngeli.

Iannuzzi, F.E. 2017. Aggirare i vincoli. La frammentazione del lavoro nell'industria dell'ospitalità veneziana. Sociologia del lavoro, 146, pp. 139-155.

Jacobs, D., Rea, A., Teney, C., Callier, L et Lothaire, S. 2009. L'ascenseur social reste en panne. Bruxelles: Fondation Roi Baudoin.

Jubany, O. and Perocco, F. (eds.) 2015. Vulnerable Workers in Times of Social Transformations: Discrimination and Participation of Young and Older Workers, and Social Dialogue Stances. Venice: Edizioni Ca' Foscari.

Lagrange, H. e Oberti, M. 2006. La rivolta delle periferie. Milano: Mondadori. Lefebvre, H. 1974. The social production of space. Oxford: Blackwell. 
Low, S. M. 2003. Embodied Space(s). Anthropological Theories of Body, Space, and Culture. Space and Culture, 6,(1), pp. 9-18.

Mancini, L. 2016. Introduction. Sociologia del diritto, 2, pp. 1-10.

Marrone, V. 2012. Spazio architettonico, spazio sociale e benessere familiare. Una analisi esplorativa. Sociologia e politiche sociali, 3, pp. 155-179.

Marzadro, M. 2010. La Cita: da complesso edilizio a quartiere urbano. Fondazione Gianni Pellicani: Venezia http://www.fondazionegiannipellicani.it/

Massey, D., 2005, For Space, Sage, London

Murie, A. 2005. The Dynamics of Social Exclusion and Neighbourhood Decline: Welfare Regimes, Decommodification, Housing, and Urban Inequality. In: Y. Kazepov, ed. Cities of Europe: Changing Contexts, Local Arrangements, and the Challenge to Urban Cohesion, pp. 151-169. Oxford: Blackwell.

Overman, H.G. 2002. Neighbourhood Effects in Large and Small Neighbourhoods Urban Studies, (39)1, pp. 117-130.

Park, R.E. and Burgess, E.W. 1928. The City. Suggestions for the Investigation of human Behavior in the Urban Environment. London: University of Chicago Press.

Perocco, F. 2012. Trasformazioni globali e nuove diseguaglianze. Il caso italiano. Milano: FrancoAngeli.

Pezzi, M.G. e Punziano, G. 2017. La categoria di "distanza" come proxy delle questioni ruralità, perifericità e sviluppo locale nella strategia nazionale per le aree interne, Sociologia e politiche sociali, 3, pp. 167-192.

Pilar Ortiz, G. e Cosimi, L. 2015. L'evoluzione del lavoro a tempo parziale in Spagna e Italia: una questione di genere?. Sociologia e politiche sociali, 1, pp. $125-143$.

Poggio, B. 2006. Il progetto GELSO: GEnere, Lavoro e Segregazione Occupazionale, Trento: Università degli Studi di Trento.

Queirolo Palmas, L. 2006. Prove di seconde generazioni. Milano: FrancoAngeli.

Raitano, M. 2011. "La riproduzione intergenerazionale delle diseguaglianze in Italia: istruzione, occupazione e retribuzioni", 3, pp. 345-374.

Rebughini, P. Colombo E. e Leonini L. 2017. Giovani dentro la crisi. Milano: Guerini e Associati.

Rosti L. 2006. La segregazione occupazionale in Italia. In Simonazzi, A. (a cura di) Questioni di genere, questioni di politica. Trasformazioni economiche e sociali in una prospettiva di genere. Milano: Carocci.

Saraceno C., Sartor, N. e Sciortino G. 2013. Stranieri e diseguali. Le diseguaglianze nei diritti e nelle condizioni di vita degli immigrati. Bologna: Il Mulino.

Sampson, R.J., Morenoff, J.D. and Gannon-Rowley, T. 2002. “Assessing 'Neighborhood Effects': Social Processes and New Directions in Research". Annual Review of Sociology, 28, pp. 443-478. 
Sayad, A. 1999. La double absence: des illusions de l'émigré aux souffrances de l'immigré. Paris: Editions du Seuil.

Schizzerotto, A., Trivellato, U. e Sartor, N. (a cura di). 2011. Generazioni disuguali. Le condizioni di vita dei giovani di oggi e di ieri:un confronto. Bologna: il Mulino.

Servizi Sociali di Marghera. 2012. Rapporto sui Servizi Sociali. Anno 2012. Venezia: Municipalità di Marghera.

Servizi Sociali di Mestre. 2014. Rapporto sui Servizi Sociali. Anno 2014. Venezia: Municipalità di Mestre Centro.

Shildrick, T. 2006. Youth culture, subculture and the importance of neighbourhood. Young, 14(1), pp. 61-74.

Storato, G. 2014. Vivere sulla soglia. I giovani adulti della banglatown, in Della Puppa, F. e Gelati, E. 2015. Alte Ceccato. Una banglatown nel nordest. Trento: Professionaldreamers.

Stravides, S. 2010. Towards the City of Threshold. Trento: Professionaldreamers.

Turner, B. 1994. Postmodern culture/modern citizens. In B. Van Steenbergen ed, The condition of citizenship, pp. 153-167. London: Sage.

Villa, M. 2016. "The transformative role of the social investment welfare state towards sustainability. Criticisms and potentialities in fragile areas", Sociologia e politiche sociali, 3, pp. 29-49.

Wacquant, L. 1995. Red belt, black belt. In E. Mingione ed, Urban poverty and underclass, pp. 234-274. London: Blackwell.

- 2007. Urban Outcast. Berkely: University of California.

Walby, S., Armstrong, J., \& Strid, S. 2012. Intersectionality: Multiple Inequalities in Social Theory. Sociology, 46(2), pp. 224-240.

Wilson,W.J. 1987. The Truly Disadvantaged: The Inner City, the Underclass, and Public Policy. Chicago: University of Chicago Press.

Woods, R.A. 1914. The Neighborhood in Social Reconstruction. The American Journal of Sociology, XIX(5), pp. 576-591.

Worth, N. 2015. "Youth, Relationality, and Space: Conceptual Resources for Youth Studies from Critical Human Geography", in Wyn J., Cahill H. (eds.), Handbook of Children and Youth Studies, Springer, Singapore, pp. 343-354.

Yuval-Davis N. 2011, The Politics of Belonging. Intersectional Contestations, Sage, London.

- 2006. Intersectionality and Feminist Politics. European Journal of Women's Studies,13(3), 193-209.

Zanfrini, L. 2011. Sociologia delle diseguaglianze. Bologna: Zanichelli. 